EDITORIAL

\title{
Can we trigger an acute coronary syndrome?
}

R A Kloner

Heart 2006;92:1009-1010. doi: 10.1136/hrt.2005.086652

While both physical exertion and anger can contribute to acute coronary syndromes, these triggers differ in their clinical and sociodemographic correlates

$\mathrm{T}$ here is an expanding literature that suggests that acute myocardial infarction and death from coronary artery disease can be triggered by a number of natural as well as man-made triggers. Natural triggers include circadian fluctuations (with an increase in myocardial infarction and cardiac death in the morning associated with the first few hours after the wake-up time ${ }^{1}$ ), seasonal fluctuations (with an increase in cardiac events during the winter months) ${ }^{2}$ and natural disasters (such as earthquakes ${ }^{3}$ and blizzards).

\section{MAN-MADE TRIGGERS}

Man-made triggers have been studied in detail and include heavy and moderate physical activity, emotional upset $^{4}$ (including anger, as described in the accompanying manuscript by Strike et $\left.a l^{5}\right)$, overeating, lack of sleep, sexual activity, and the use of cocaine and other illicit substances. ${ }^{4}$ Certain man-made events may trigger cardiac events. For example, we recently observed that there was an increase in ischaemic heart disease death rates during the millennium celebration of the New Year versus other New Years' celebration. ${ }^{6}$ Another report of an increase in cardiac events induced by a man-made event was the increase in acute myocardial infarction and sudden death reported in Israel at the time of Iraqi missile attacks. ${ }^{7}$ We were surprised to learn that some man-made unnatural disasters that we hypothesised might be associated with an increase in death due to ischaemic heart disease were not. An example is the terrorist attacks of 11 September 2001. A review of death certificate data from New York City did not reveal an increase in death from ischaemic heart disease on the day of the attacks compared to that same day during previous years. ${ }^{8}$ We cannot rule out the possibility that some deaths in the twin towers were cardiac in nature-it may have been that these events were masked by death from trauma/fire. We cannot rule out the possibility that there will be long term health problems because of the attack. However, simply watching the news about 9/11 on television was apparently not a sufficient stressor to induce an increase in cardiac deaths in the New York City population. The riots in Los Angeles following the Rodney King conviction also were not associated with an increase in cardiac events. ${ }^{9}$
Triggers that are associated with an increase in acute myocardial infarction and ischaemic heart disease death may share certain common mechanisms. They are in general associated with increases in sympathetic activity and catecholamine release-the so called "fight or flight" response. ${ }^{5}$ Both physical and emotional stress may precipitate sympathetic activity and catecholamine release leading to a number of haemodynamic changes, including an increase in heart rate, blood pressure, vascular resistance, and ventricular contractility. These factors can increase shear stress of blood against a vulnerable atherosclerotic plaque, contributing to rupture of the plaque and subsequent myocardial infarction. In addition, these haemodynamic factors can increase oxygen demand (increase heart rate, contractility, and blood pressure) at the same time they reduce oxygen supply (an increase in coronary resistance or frank coronary spasm; an increase in platelet aggregability). Furthermore, stimulation of the sympathetic nervous system may trigger arrhythmias with or without concomitant ischaemia. ${ }^{10}$

\section{PHYSICAL EXERTION AND ANGER}

The present paper by Strike et al extends our knowledge of two specific known triggersphysical exertion and anger. Their work confirms previous reports by verifying that both physical exertion and anger contributed to acute coronary syndromes (primarily ST elevation acute myocardial infarction), but each trigger differed somewhat in their clinical and sociodemographic correlates. Specifically, acute myocardial infarction associated with physical exertion was more likely to occur in patients who were not socially deprived. These patients were more likely to develop a Q wave ST elevation myocardial infarction with greater enzyme release compared to patients who were sedentary before their coronary events. These patients were also more likely to have a higher GRACE score-which includes a history of congestive heart failure. Coronary events in these patients typically occurred in the afternoon and were unlikely to be associated with premonitory symptoms.

When anger was the trigger the patients tended to be younger, have lower socioeconomic status, have premonitory symptoms, and were more likely to present with ST elevation myocardial infarction rather than non-ST elevation myocardial infarction or unstable angina. Troponin release was not as great in this group. There was a trend towards more hypertension in patients who had anger versus exertion as a trigger.

Thus each of these triggers was associated with a different clinical presentation and 
socioeconomic patient profile. These observations suggest but do not prove that the two types of triggers work through two different final mechanisms of action.

As the authors point out, physical exertion may have stimulated plaque rupture (with a larger Q wave infarct and more troponin release). Perhaps anger stimulated transient coronary vasospasm in some patients without plaque rupture (ST elevation myocardial infarction but not as many Q wave myocardial infarctions, less enzyme release, and less heart failure). Additional studies will be needed to verify this concept-but the concept that different triggers may be associated with different clinical presentations of acute coronary syndromes is a novel finding and the authors are to be congratulated.

It would be important to determine whether there are also unique profiles for clinical presentation of acute coronary syndromes and socioeconomic differences in patients who have other types of triggers-overeating, lack of sleep, sexual activity, cocaine and others-or in those patients who do not have identifiable triggers.

\section{PREVENTING THE TRIGGER}

An important question in the field of triggers for myocardial infarction is whether the incidence of acute coronary syndromes can be further reduced by directly trying to prevent the trigger. For example, are people who are prone to anger, but have had anger management intervention, less likely to have a coronary event compared to those who have not had anger management counselling? Would a larger study in which a systematic physical training programme with emphasis on a warm up period before vigorous exercise reduce cardiac events in those patients who then exercise vigorously? Would a programme aimed at improving people's socioeconomic status reduce the incidence of coronary events? Can certain medicines reduce the triggering phenomenon? It does appear that certain pharmacologic agents may modulate cardiac events associated with the wake-up time. Long acting $\beta$ blockers and calcium channel blockers can decrease the frequency of morning episodes of ischaemia, based on ambulatory ECG monitoring. ${ }^{11}{ }^{12}$ The morning peak of acute myocardial infarction was notably blunted by aspirin-presumably related to a reduction in platelet aggregation. ${ }^{13}$ Statins are thought to stabilise the vulnerable atherosclerotic plaque and hence may prevent triggered rupture of the plaque.

There is a need for more outcome trials aimed at specifically reducing known triggers of acute myocardial infarction. Eliminating myocardial infarcts associated with triggers will eliminate a substantial percentage of myocardial infarcts. Based on the present study, ${ }^{5}$ eliminating acute coronary syndromes associated with anger and physical exertion could eliminate $17 \%$ and $10 \%$ of such events, respectively.

Competing interest statement: There is no competing interest.

\section{REFERENCES}

1 Rocco MB, Barry J, Campbell S, et al. Circadian variation of transient myocardial ischemia in patients with coronary artery disease. Circulation 1987:73:395-400

2 Kloner RA, Poole WK, Perritt RL, et al. When throughout the year is coronary death most likely to occur? A 12-year population based analysis of more than 222,000 cases. Circulation 1999;100:1630-4.

3 Leor J, Poole WK, Kloner RA, et al. Sudden cardiac death triggered by an earthquake. N. Engl J Med 1996;334:413-19.

4 Muller JE, Abela GS, Nesto RW, et al. Triggers, acute factors, and vulnerable plaques: the lexicon of a new frontier. J Am Coll Cardiol 1994;23:809-13.

5 Strike PC, Perkins-Porras L, Whitehead DL, et al. Triggering of acute coronary syndromes by physical exertion and anger: clinical and sociodemographic characteristics. Heart 2006;92:1035-40.

6 Poole WK, Chi JS, Walton JD, et al. Increased cardiovascular mortality associated with the turn of the millennium in Los Angeles County, California. $J$ Epidemiol Community Health 2005:59:205-6.

7 Meisel SR, Kutz I, Dayan Kl, et al. Effect of Iraqi missile war on incidence of acute myocardial infarction and sudden death in Israeli civilians. Lancet $1991 ; 338: 660-1$

8 Chi JS, Poole WK, Kandefer SC, et al. Cardiovascular mortality in New York City after September 11, 2001. Am J Cardiol 2003;92:857-61.

9 Birnbaum Y, Kloner RA, Perritt R, et al. Atherosclerotic cardiovascular mortality during the 1992 riots in Los Angeles. Am J Cardiol 1997;79:1155-8.

10 Kloner RA. Natural and unnatural triggers of myocardial infarction. Progress in Cardiovascular Disease (in press).

11 Deedwania PC, Carbajal EV. Role of beta blockade in the treatment of myocardial ischemia. Am J Cardiol 1997;80:23J-8J.

12 Deanfield JE, Detry J-M, Lichtlen PR and the CAPE Study Group. Amlodipine reduces transient myocardial ischemia in patients with coronary artery disease, double-blind circadian anti-ischemic program in Europe (CAPE trial). J Am Coll Cardiol 1994;24:1460-7.

13 Ridker PM, Manson JE, Buring JE, et al. Circadian variation of acute myocardial infarction and the effect of low dose aspirin in a randomized trial of physicians. Circulation 1980;82:897-902.

\section{STAMPS IN CARDIOLOGY}

\section{Thrombosis}

$\mathrm{T}$ hrombosis has not specifically featured on the world's postage stamps previously. The $\$ 10$ stamp from the Dominican Republic is the only stamp therefore issued on this topic. It was released to mark the $15^{\text {th }}$ International Congress on Haemostasis and Thrombosis held in 1997. There is an error on the stamp printing with an upward shift of the yellow colour.

M K Davies A Hollman

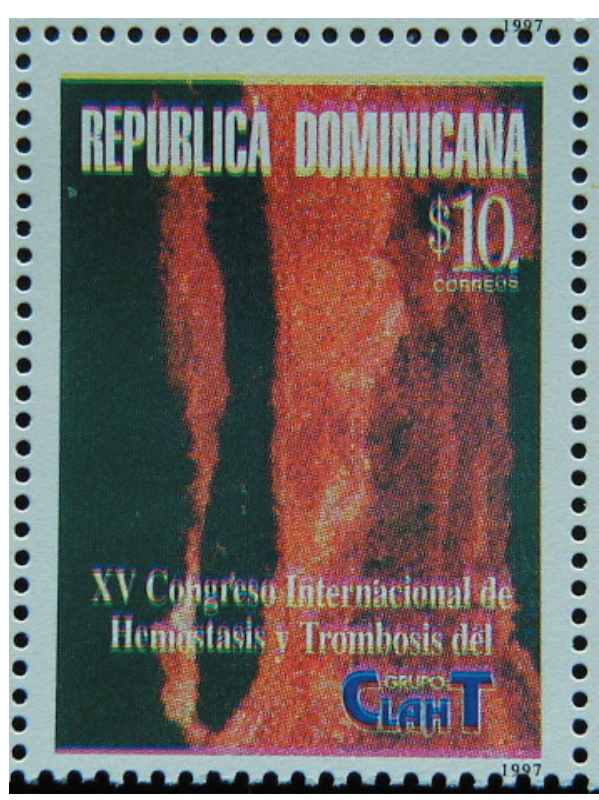

